\title{
Kinematic Characteristics of Reaching Movements in Preterm Children with Cerebral Palsy
}

\author{
JOLANDA C. VAN DER HEIDE, JOHANNA M. FOCK, BERT OTTEN, \\ ELISABETH STREMMELAAR, AND MIJNA HADDERS-ALGRA

\begin{abstract}
Department of Neurology [J.C.v.d.H., J.M.F., M.H.-A.], 9713 GZ University of Groningen, Groningen, The Netherlands; Department of Human Movement Sciences [B.O.], 9713 AV University of Groningen, Groningen, The Netherlands; and Department of Paediatrics [E.S.], 9713 GZ University Hospital
\end{abstract}

Groningen, Groningen, The Netherlands

\begin{tabular}{|c|c|}
\hline \multicolumn{2}{|c|}{ ABSTRACT } \\
\hline $\begin{array}{l}\text { Kinematic characteristics of reaching movements of the dom- } \\
\text { inant arm were assessed in } 51 \text { sitting preterm children who were } \\
\text { aged } 2-11 \text { y and had cerebral palsy }(\mathrm{CP}) \text {, including } 33 \text { with } \\
\text { spastic hemiplegia and } 18 \text { with bilateral } \mathrm{CP} \text { (Bi-CP). Reference } \\
\text { data of } 29 \text { typically developing children were present. The results } \\
\text { indicated that the quality of reaching movements from the dom- } \\
\text { inant arm of children with CP was significantly worse than that } \\
\text { of typically developing children. This held true in particular for } \\
\text { the children with Bi-CP. For example, reaching movements of } \\
\text { children with CP took more time and consisted less often of one } \\
\text { movement unit. The quality of reaching was related to the } \\
\text { severity of lesion present on the neonatal ultrasound scan of the } \\
\text { brain, the severity of motor disorder, the degree of spasticity, and }\end{array}$ & $\begin{array}{l}\text { the ability to perform activities of daily life. The last indicates } \\
\text { that movements of the dominant arm in children with spastic } \\
\text { hemiplegia and Bi-CP deserve clinical attention. (Pediatr Res } \\
\text { 57: 883-889, 2005) } \\
\text { Abbreviations } \\
\text { Bi-CP, bilateral cerebral palsy } \\
\text { CP, cerebral palsy } \\
\text { GMFCS, Gross Motor Function Classification System } \\
\text { MU, movement unit } \\
\text { PEDI, Pediatric Evaluation of Disability Inventory } \\
\text { PVL, periventricular leukomalacia } \\
\text { SH, spastic hemiplegia }\end{array}$ \\
\hline
\end{tabular}

During daily life, we make many reaching movements. Reaching movements in healthy adults are characterized by a bell-shaped velocity profile that consists of one acceleration and one deceleration (1). It takes a long time, however, before reaching movements have this adult configuration. The first reaching movements, which emerge at $\sim 3-4$ mo of age, are characterized by variation and irregular, zig-zag-like trajectories $(2,3)$. During the following months, the reaching movements rapidly become more regular and smooth. Thereafter, fine-tuning of reaching takes many years. This is reflected in the development of the kinematic characteristics of reaching. The reaching movements become faster, straighter, and smoother (4-10). The increase in smoothness of the reaching movements is due to a decrease of the corrections of the movement path. These corrections are termed movement units

Received January 12, 2004; accepted October 13, 2004.

Correspondence: Prof. Dr. Mijna Hadders-Algra, University Hospital Groningen, Developmental Neurology, Hanzeplein 1, 9713 GZ Groningen, The Netherlands; e-mail: m.hadders-algra@med.rug.nl.

This study was supported by the Johanna KinderFonds (grant 19990021), the Dr. W.M. Phelps-Stiching voor Spastici (grant 99.058), and the Gratama Stichting/Groninger Universiteits Fonds.

DOI: 10.1203/01.PDR.0000157771.20683.14
(MUs). They are submovements of the reaching movement and are determined with the help of peaks in the velocity profile of the reaching hand $(2,4)$. Reaching movements of infants who are starting to reach consist of 3-7 MUs, whereas those at $12 \mathrm{y}$ have an adult configuration of 1 MU (1-3,7). In parallel, an increase of the relative length and duration of the first MU, the so-called transport MU, is seen so that it gradually covers a larger proportion of the approach to the target $(2,4)$.

Relatively little is known about the kinematics of reaching in children with cerebral palsy (CP). Some studies evaluated the difference in kinematic characteristics between the most and least affected arm in individual who had spastic hemiplegia and were aged 4-20 y (11-16). The studies indicated that the most involved arm had longer reaching durations, a lower $\mathrm{V}_{\max }$, and more MUs than the least involved arm. However, no comparison with performance of typically developing age-matched controls was made. Two other studies assessed kinematics of reaching in children with spastic quadriplegia with the aim of evaluating the effect of an intervention program $(17,18)$. From these studies, it can be gathered that children who were aged 7-12 y showed reaching movements that in general consisted of multiple MUs (mean value 3.7). This performance differs 
substantially from that of typically developing children at this age, who mainly exhibit reaching movements of one MU [mean value $1.2(7)]$.

The aim of our study was to evaluate the kinematic characteristics of reaching movements of preterm children with $\mathrm{CP}$. To this end, we studied 58 children who have spastic CP and were aged 2-11 y, 34 having a spastic hemiplegia ( $\mathrm{SH})$ and 24 a bilateral form of spastic $\mathrm{CP}$ (Bi-CP), and 29 age-matched typically developing children. The children made reaching movements with their dominant arm, which means for the children with SH the least affected arm. We deliberately chose the dominant arm, as we were interested in reaching during daily life activities. Kinematics of the wrist was recorded while the children made reaching movements in a sitting position. The following questions were addressed: 1 ) Do the kinematics of reaching of children with $\mathrm{CP}$ differ from those of typically developing children? 2) If they differ, then are the kinematics of reaching of children with CP influenced by $a$ ) type of CP (SH versus $\mathrm{Bi}-\mathrm{CP}), b)$ age, $c$ ) gestational age at birth, $d$ ) severity of disability, and $e$ ) uni- or bilateral nature of the lesion on the neonatal ultrasound scan of the brain and $f$ ) the degree of spasticity of the reaching arm as measured by the modified Ashworth scale (19)? 3) Is reaching performance in children with $\mathrm{CP}$ related to the clinical severity of the disability to functional performance in daily life as measured by the Pediatric Evaluation of Disability Inventory (PEDI) (20)?

\section{METHODS}

Participants. The study group consisted of 58 children with CP, aged 2-11 y. Thirty-four had a SH, 17 a right-sided one and 17 a left-sided one, and 24 had Bi-CP. In seven children, spasticity was associated with dyskinesia, and in five, spasticity was associated with ataxia. Children with a severe visual impairment and those who were not able to reach out for an object were excluded from the study. All but one of the children with SH were able to walk without assistive devices; they had a level I $(n=33)$ or a level IV $(n=1)$ disability according to the Gross Motor Function Classification System (GMFCS) (21). In the group of children with Bi-CP, nine children could walk without assistive devices, 10 could walk with the help of assistive mobility devices (GMFCS level III), and five had limited self-mobility (GMFCS level IV-V).

All children with $\mathrm{CP}$ were born preterm. Gestational age and weight at birth did not differ for the groups of children with $\mathrm{SH}$ [gestational age median (range): 29 wk (25-33 wk); birth weight median (range): $1065 \mathrm{~g}(620-1915$ g)] and those with Bi-CP [gestational age median (range): 28.5 wk (26-32

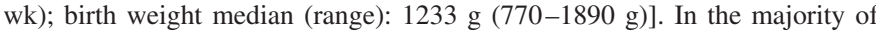
children, serial neonatal brain ultrasound scans had been made with a highresolution $7.5-\mathrm{MHz}$ transducer until 1 mo postterm. After this age, a 5-MHz transducer was used. The children were scanned during the first weeks after birth at various intervals (ranging from $3 \mathrm{~d}$ to $2 \mathrm{wk}$ ). Periventricular hemorrhages and leukomalacia (PVL) in the preterm children were classified according the methods of Levene et al. (22) and De Vries et al. (23), respectively. The severity of the brain lesions was classified as no lesion, as mild in the case of the presence of a grade 1 or 2 PVL and/or hemorrhage grade 1 or 2 , or as severe in the case of a PVL grade 3 and/or hemorrhage grade 4. Another variable addressed the unilateral or bilateral nature of the brain lesion.

The reference group consisted of 29 children who were born at term with a typical motor development. Nine children of the typically developing group were $2-4$ y, 10 children were 5-7 y, and another 10 were 8-11 y (10). All parents gave informed consent. The Medical Ethics Committee of the University Hospital Groningen approved the study.

Procedure. The majority of children sat on a table without back and foot support. The children who were not able to sit independently for the duration of the experiment received additional support. Back and foot support was provided in five of the 12 children with severe CP. Foot support only was given to three children with severe $\mathrm{CP}$ and one young child with moderately severe CP. Foot support consisted of placing the child's feet on a low bench. Trunk support was provided by the hands of a research assistant, which were placed on the back side of the child's pelvic girdle. The examiner presented an attractive small puppet (length $6 \mathrm{~cm}$, width $2 \mathrm{~cm}$ ) in the midline at arm's-length distance of the subject. The instruction was to grasp the puppet with the dominant hand at a natural, self-paced speed. The dominant hand was defined as the hand with which the child preferred to write or draw. Ten to 20 trials were performed. Before testing, the children carried out some exercise trials.

Movements were recorded kinematically with an ELITE opto-electronic movement recording system (BTS, Milan, Italy) in a four-camera configuration at a sampling frequency of $50 \mathrm{~Hz}$. A reflective marker was placed at the dominant hand on the styloid process of the radius. Sampling of the kinematic data started a few seconds before toy presentation and lasted for 8-12 s, depending on the reaching velocity of the child. Kinematic recording in young children is technically cumbersome and is associated with a considerable loss of data. Children were included in the analyses only when at least three appropriate kinematic trials could be achieved (Table 1). One child with SH of 3 y and six children with Bi-CP failed the three-trials criterion (the child with SH had a GMFCS level I; of the children with Bi-CP, one had level I, one had level II, two had level III, and two had level IV). Also, three typically developing children did not meet the three-trials criterion, two in the youngest age group and one child who was $5 \mathrm{y}$ of age. In addition to the kinematic analysis of the reaching movements, postural control was assessed by means of multiple surface electromyograph recordings (24) and kinematics (24a). The postural data are not included in the present data analyses.

The whole session was recorded on video. After the reaching sessions, neurologic condition, functional performance, and degree of spasticity of the children were evaluated. On the basis of the neurologic examination, three classes of severity of the disorder were distinguished: mild, moderate, and severe, indicating that posture, motility, muscle tone, and reflexes were affected to a limited, moderate, and severe extent, respectively (Table 2). Functional performance in self-care, transfers, and social abilities was assessed during a telephone interview a few days after the kinematic evaluation by means of the standardized PEDI (20) Table 2). Degree of spasticity of the biceps brachii muscle of the dominant arm was assessed with the help of the modified Ashworth scale (19).

Data analysis. The video recordings were used to select reaching movements with the dominant arm that ended in successful grasping of the object during which the child was in an appropriate state of attention. The latter meant that the children had to be in a quiet, attentive behavioral state and looking at the presented object. Such a selection procedure was needed in particular in the youngest children.

Arm movement onset was defined as the moment at which the velocity of the wrist increased $>5 \%$ of peak velocity, whereas the moment at which wrist velocity declined to $5 \%$ of peak velocity at the farthest distance in space relative to the beginning of the movement was considered as the end of the movement. Off-line kinematic analysis was carried out with the help of the software package DataMonster 2.0 (25) (E. Otten, Department of Human Movement Sciences, University of Groningen). The data were filtered using a low-pass filter of $3 \mathrm{~Hz}$ (zero time-lag filter). In the kinematic analysis, only trials with a clearly demarcated start and stop were included (Table 1). We used the following parameters to describe the reaching movements: 1) reaching duration, i.e. the total time needed from the start of the reaching movement until contact with the puppet; 2) maximum reaching velocity, as revealed by the $\mathrm{V}_{\max }$ of the wrist; 3) index of curvature of the reaching movement, as indicated by the ratio of the actual length of the reaching path and the length of the straight line between starting and stopping position [expressed in \% (26)]; 4) the proportion of trials during which the reaching movement consisted of one MU; and 5) the length of the first MU (the transport MU) relative to total movement path (in \%). An MU consisted of one acceleration and one deceleration in the velocity profile of the wrist marker $(2,4)$.

Statistics. Statistical analyses were performed using the computer package SPSS (version 10.1). The majority of analyses were performed on child level, meaning that for the kinematic variables, first the child's mean value was calculated on the basis of which further analyses were performed. The data on the level of the individual child had nonnormal distributions. Therefore, we used for the evaluation of the effect of type of $\mathrm{CP}$ (SH or Bi-CP), effect of age, and the presence of additional back or foot support the nonparametric MannWhitney test. To establish the influence of the uni- or bilateral nature of the brain lesion on the ultrasound scan on the kinematic parameters of postural control, we used the Kruskal-Wallis test. To be able to evaluate the effect of age while taking into account the effect of group and the severity of disability, we used ANOVAs. The post hoc Bonferroni test was used to identify the loci of significance for these analyses. We are aware that the parametric ANOVA analyses in fact are nonoptimal, but the ANOVAs offered the only solution to get some idea on the aggregate effect of multiple factors on kinematic outcome. For the relation between clinical characteristics, such as the PEDI, gestational age, and the severity of brain lesion and the kinematic parameters, we calculated Spearman $\rho$. To be able to differentiate between the effects of 
Table 1. Number of trials per individual with successful kinematic recording

\begin{tabular}{clcc}
\hline & & \multicolumn{2}{c}{ No. of trials } \\
\cline { 3 - 4 } Age & \multicolumn{1}{c}{ Group } & Median & Range \\
\hline \multirow{2}{*}{$2-4$ y } & Children with SH $(n=8)$ & 7 & $4-10$ \\
& Children with Bi-CP $(n=4)$ & 6 & $4-9$ \\
& Typically developing children $(n=7)$ & 4 & $3-9$ \\
$5-7$ y & Children with SH $(n=12)$ & 5 & $3-9$ \\
& Children with Bi-CP $(n=7)$ & 4 & $3-10$ \\
& Typically developing children $(n=9)$ & 9 & $5-11$ \\
$8-11$ y & Children with SH $(n=13)$ & 6 & $3-10$ \\
& Children with Bi-CP $(n=7)$ & 6 & $5-11$ \\
& Typically developing children $(n=10)$ & 11 & $4-13$ \\
\hline
\end{tabular}

gestational age [born before $28 \mathrm{wk}$ or $\geq 28 \mathrm{wk}$ (24)] versus severity of brain lesion and the effects of degree of spasticity of the reaching arm versus severity of brain lesion on the kinematic parameters, we performed two runs of multiple regression analyses. In general, we considered differences and correlations with $p<0.05$ as statistically significant. The exceptions to this rule were the ANOVAs for which many comparisons were made. There differences with $p<0.01$ were considered as statistically significant to decrease the risk of alluding significance to chance findings.

\section{RESULTS}

Kinematics of reaching. Figure 1 displays examples of three reaching movements of a typically developing child, two children with SH (one with and one without a severe brain lesion), and two children with Bi-CP (one with and one without a severe brain lesion). Children with $\mathrm{SH}$ and Bi-CP had significantly longer reaching durations than typically developing children (median values: typically developing $1.0 \mathrm{~s}$; SH $1.3 \mathrm{~s}$; Bi-CP $1.3 \mathrm{~s} ; p<0.01 ;$ Fig. 2). They also exhibited significantly less often than the typically developing children reaching movements that consisted of one MU (median values: typically developing 80\%; SH 67\%; Bi-CP 50\%; $p<0.01$; Fig. 2). Children with Bi-CP also exhibited less straight (median values: 108 versus $105 \% ; p<0.01)$ and slower reaching movements than the typically developing children (median values: 0.66 versus $0.90 \mathrm{~m} / \mathrm{s} ; p<0.01$ ). In the children with $\mathrm{Bi}-\mathrm{CP}$, the proportion of the reaching movement covered by the transport MU was smaller than that in the typically developing children (median values: 90 versus $98 \% ; p<0.01$ ). Similar differences between children with $\mathrm{SH}$ and typically developing children were absent.

Postnatal age affected the kinematics of reaching. This was especially true for the typically developing children. Typically developing 2- to 4-y-olds had a lower peak velocity, fewer trials with one MU, less straight reaching movements, and reaching movements of which a lesser proportion was covered by the transport MU than typically developing 8- to 11-y-olds (median values, respectively: 0.62 versus $0.94 \mathrm{~m} / \mathrm{s}, 67$ versus $88 \%, 108$ versus $103 \%$, 91 versus $99 \% ; p<0.01)$. In the children with $\mathrm{SH}$, age affected only the index of curvature (5to 7 -y-olds $108 \%$ versus 8 - to 11 -y-olds $104 \% ; p<0.01$ ). In the group of children with $\mathrm{Bi}-\mathrm{CP}$, age did not affect the kinematics of reaching.

Within the group of children with $\mathrm{CP}$, reaching duration, the proportion of reaches with one $\mathrm{MU}$, and the proportion covered

Table 2. Clinical data of the children with $C P$

\begin{tabular}{|c|c|c|c|c|c|c|}
\hline & \multicolumn{3}{|c|}{$\mathrm{SH}$} & \multicolumn{3}{|c|}{$\mathrm{Bi}-\mathrm{CP}$} \\
\hline Mild & 5 & 9 & 10 & 2 & 2 & 2 \\
\hline Moderate & 2 & 3 & 3 & 1 & 3 & 1 \\
\hline Severe & 1 & 0 & 0 & 1 & 2 & 4 \\
\hline Median & 49.5 & 67 & 72 & 36 & 62 & 60 \\
\hline Range & $23-68$ & $51-74$ & $60-74$ & $30-55$ & $51-66$ & $25-74$ \\
\hline \multicolumn{7}{|l|}{ Fsmob } \\
\hline Median & 54 & 59 & 61 & 45 & 42 & 34 \\
\hline Range & $10-58$ & $53-61$ & $58-61$ & $28-53$ & $18-59$ & $20-61$ \\
\hline \multicolumn{7}{|l|}{ Fssoc } \\
\hline Range & $3-28$ & $22-40$ & $33-40$ & $7-22$ & $24-38$ & $4-40$ \\
\hline \multicolumn{7}{|l|}{ Camob } \\
\hline Median & 32.5 & 35 & 35 & 27 & 35 & 17 \\
\hline Range & $5-35$ & $34-35$ & $33-35$ & $21-35$ & $10-35$ & $5-35$ \\
\hline \multicolumn{7}{|l|}{ Casoc } \\
\hline Median & 14 & 20.5 & 22 & 18 & 24 & 24 \\
\hline Range & $5-20$ & $8-25$ & $20-25$ & $14-22$ & $19-25$ & $20-25$ \\
\hline \multicolumn{7}{|l|}{ Total score } \\
\hline Median & 218.5 & 275.5 & 292 & 189 & 253 & 225 \\
\hline Range & $91-260$ & $218-301$ & $269-301$ & $157-240$ & $184-281$ & $135-300$ \\
\hline
\end{tabular}

fssc, functional scale self-care; fsmob, functional scale mobility; fssoc, functional scale social function; casc, caregiver assistance self-care; camob, caregiver assistance mobility; casoc, caregiver assistance social function. 
A
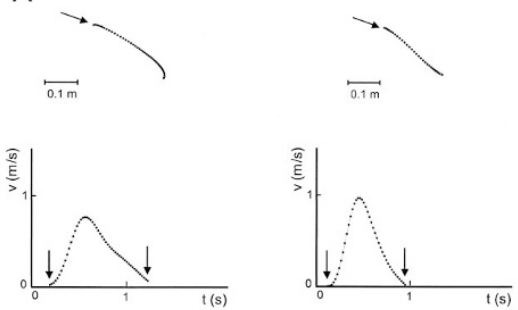

B
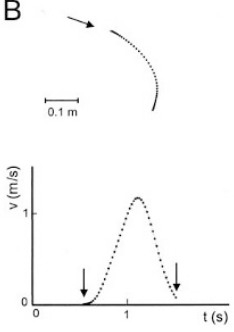

$\rightarrow$
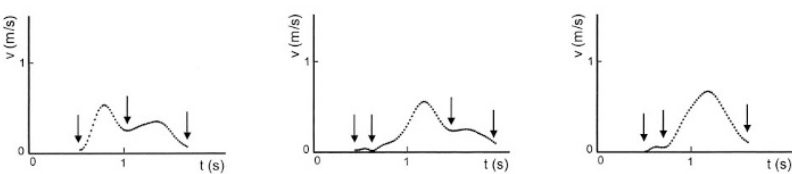

C
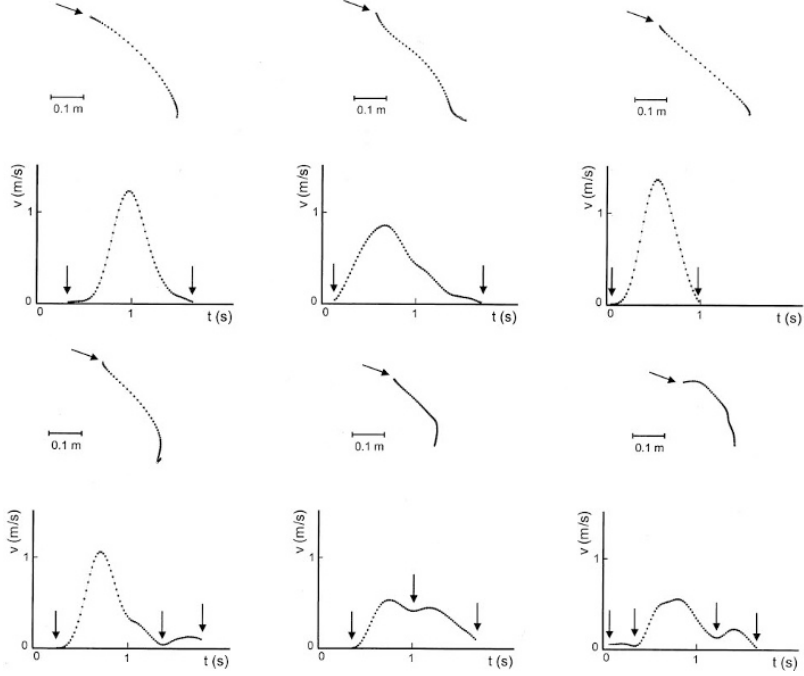

Figure 1. Reaching trajectories and velocity profiles. Typical examples of reaching movements of a typically developing child of $9 \mathrm{y}(A)$, two children with SH ( $B$; top: a child of 9 y born after 30 wk of gestation, with a bilateral located grade 2 periventricular hemorrhage and a PEDI total score of 301 ; bottom: a child of $7 \mathrm{y}$, born at $29 \mathrm{wk}$ of gestation with a bilateral periventricular hemorrhage grade 4 and PEDI total score of 294), and two children with Bi-CP ( $C$; top: a child of $10 \mathrm{y}$ born at $32 \mathrm{wk}$ of gestation with a bilateral grade 2 periventricular hemorrhage and a PEDI total score of 292; bottom: a child of $9 \mathrm{y}$ born at $30 \mathrm{wk}$ of gestation with a grade 4 periventricular hemorrhage parietally on the right side and a PEDI total score of 225). The four children with CP performed the reaching tasks without additional postural support. Each panel consists of two parts: the top row shows the reaching trajectories, the bottom row shows the velocity profile of the wrist marker of the same reaching movement. The arrows in the figures of the reaching trajectories indicate the start of the reaching movement; the dots are separated by a time window of $20 \mathrm{~ms}$. The arrows in the figures of the velocity profiles indicate the beginning and the end of an MU. The children with a severe brain lesion show less straight reaching trajectories and reaching movements that consist of more than one MU. by the transport MU were not related to the severity of the disability, but the severity of disability was related to the index of curvature of the reaching movements. Post hoc comparisons revealed that children with severe $\mathrm{CP}$ had less straight reaches than children with mild or moderate $\mathrm{CP}$ (means: mild, $106 \%$; moderate, $106 \%$; severe, $115 \%$; ANOVA $F_{2,8}=15.2, p<$ $0.01)$. For peak velocity, a main effect for severity of disability (means: mild, $0.78 \mathrm{~m} / \mathrm{s}$; moderate, $0.74 \mathrm{~m} / \mathrm{s}$; severe, $0.58 \mathrm{~m} / \mathrm{s}$; ANOVA $\left.F_{2,8}=9.4, p<0.01\right)$ and an interaction effect was found between age group and severity (ANOVA $F_{4,8}=8.5$, $p<0.01)$. Post hoc analysis showed that in children with mild or moderate forms of $\mathrm{CP}$, reaching velocity increased with increasing age, whereas it decreased with increasing age in children with severe CP. The kinematics of reaching of children who received additional postural support during testing was virtually similar to that of the children who had severe $\mathrm{CP}$ and did not receive extra support. The only marginal difference found was that the children who had severe or moderate $\mathrm{CP}$ and received additional support tended to have slower reaching movements than children who had severe $\mathrm{CP}$ and were tested without extra support (median values: 0.45 versus $0.71 \mathrm{~m} / \mathrm{s}$; $p=0.07$ ).

Neonatal characteristics and kinematics of reaching. The kinematics of reaching in children with $\mathrm{CP}$ was related to some extent to the severity of the lesion on the neonatal ultrasound scan of the brain. A more severe brain lesion was related to a lower percentage of reaches with one $\mathrm{MU}(\rho=-0.36, p<$ 0.01 ) and to the index of curvature indicating that a more severe lesion was associated with less straight reaching $(\rho=$ $0.34, p<0.05)$. In the subgroup of children with $\mathrm{Bi}-\mathrm{CP}$, the relation between the severity of the brain lesion and the proportion of reaches with one MU persisted $(\rho=-0.57, p<$ $0.05)$. In the children with $\mathrm{SH}$, the association between a more severe brain lesion and less straight reaching approached statistical significance $(\rho=0.34, p=0.05)$. When a brain lesion was present, the uni- or bilateral nature of the lesion did not affect the kinematics of reaching.

In children with $\mathrm{Bi}-\mathrm{CP}$, gestational age at birth was related to straightness of the reaching movement: children who were born at younger gestational ages reached less straight $(\rho=$ $-0.47, p<0.05)$. A similar relation was not found in the children with SH. Gestational age at birth was not related to reaching duration, peak velocity, the percentage of trials that consisted of $1 \mathrm{MU}$, and the proportion of the transport MU. Because in the children with $\mathrm{CP}$ gestational age at birth was negatively related to the severity of brain lesion $(\rho=-0.48$, $p<0.01$ ), we wondered which of the two factors (severity of brain lesion or gestational age) affected kinematics most. Multiple regression analysis revealed that in the children with $\mathrm{CP}$, severity of brain lesion had a negative effect on the percentage of reaches that consisted of one MU $(p<0.05)$ and on the index of curvature $(p=0.05)$, whereas no effect for gestational age at birth was left.

Functional measurements and kinematics of reaching. All reaching parameters - with the exception of total reaching duration-were significantly related to the scores on the PEDI (Table 3). When the children with $\mathrm{CP}$ were pooled, a higher peak velocity, a higher percentage of trials with one MU, 


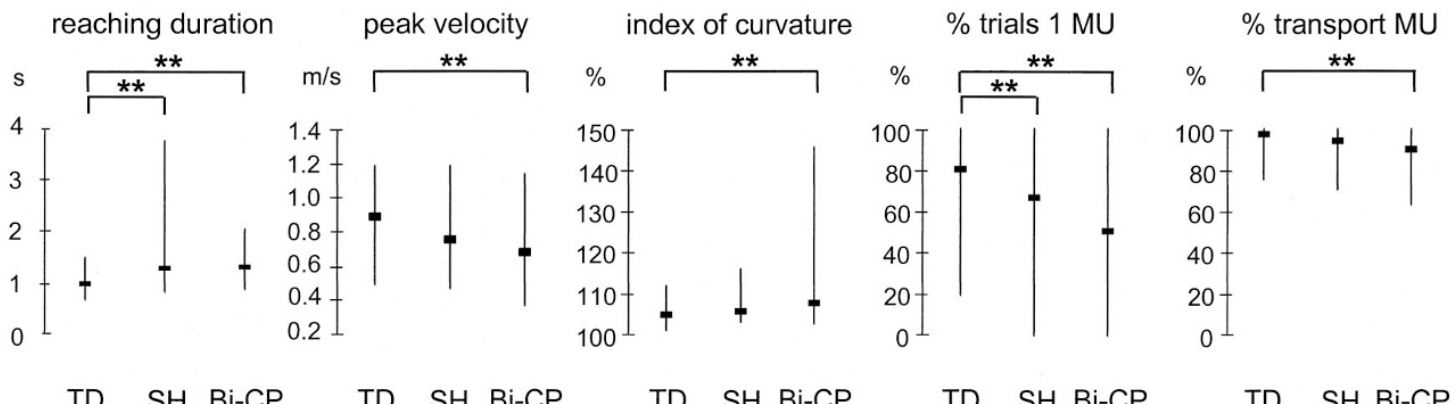

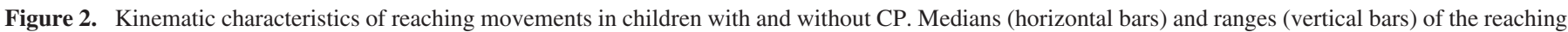
parameters. TD, typically developing. Mann-Whitney, $* p<0.05, * * p<0.01$.

straighter reaching movements, and reaching movements during which the transport MU covers a higher proportion of the movement were related to better PEDI scores. The only PEDI subscore that was not related to the kinematics of reaching was caregiver assistance on the social function domain. In the children with Bi-CP, kinematics of reaching were related stronger to PEDI scores than in the children with SH (Table 3).

Kinematics of reaching in children with $\mathrm{CP}$ was related to some extent to the degree of spasticity measured by the modified Ashworth scale. A higher degree of spasticity was related to a lower percentage of trials with one $\mathrm{MU}$ ( $\rho=-0.33, p<$ $0.05)$ and less straight reaching movements $(\rho=0.47, p<$ 0.01 ). The association between a higher degree of spasticity and less straight reaching movements was brought about by the children with $\mathrm{Bi}-\mathrm{CP}$, for whom the relationship was highly significant $(\rho=0.63, p<0.01)$. In the children with $\mathrm{SH}$, among whom only one child had a minimal degree of spasticity in the dominant arm, the degree of spasticity was not related to the kinematics of reaching. The degree of spasticity was not related to reaching duration, peak velocity, and the proportion of the transport MU. Because in the children with Bi-CP the degree of spasticity of the reaching arm was related to the severity of brain lesion ( $\rho=0.55, p<0.05$ ), we wondered which of the two factors (severity of brain lesion or spasticity) affected kinematics most. Multiple regression analysis revealed that in the children with Bi-CP especially, the severity of the brain lesion affected the kinematics of reaching: it had a significant negative effect on the percentage of reaches that consisted of one MU $(p<0.05)$, and it tended to affect the index of curvature $(p=0.08)$, whereas no effect for the degree of spasticity was left.

\section{DISCUSSION}

The present study demonstrated that the kinematic quality of reaching movements of the dominant arm of preterm children with $\mathrm{SH}$ and $\mathrm{Bi}-\mathrm{CP}$ was significantly worse than that of age-matched typically developing term control subjects. This finding has a clinical importance as we also demonstrated that the quality of reaching was related to the ability to perform activities of daily life.

Before attributing all differences between the children with $\mathrm{CP}$ and the typically developing children to the presence or absence of $\mathrm{CP}$, it is good to keep in mind that also the

Table 3. Spearman rank correlations between the subscales and total scores of the PEDI and the kinematic parameters for the children with $C P$

\begin{tabular}{|c|c|c|c|c|c|c|c|}
\hline & PEDI fssc & PEDI fsmob & PEDI fssoc & PEDI casc & PEDI camob & PEDI casoc & PEDI total score \\
\hline \multicolumn{8}{|l|}{ All children with $\mathrm{CP}$} \\
\hline Reaching duration & -0.02 & -0.03 & 0.11 & 0.01 & -0.19 & 0.01 & -0.02 \\
\hline Peak velocity & 0.40 & 0.44 & 0.32 & 0.48 & 0.26 & 0.11 & 0.43 \\
\hline$\%$ Trials $1 \mathrm{MU}$ & 0.34 & 0.23 & 0.16 & 0.33 & 0.38 & 0.10 & $\mathbf{0 . 3 3}$ \\
\hline$\%$ Transport MU & 0.43 & 0.26 & 0.29 & 0.43 & 0.39 & 0.12 & 0.41 \\
\hline \multicolumn{8}{|l|}{ Children with SH } \\
\hline Index of curvature & -0.40 & -0.33 & -0.32 & -0.37 & -0.41 & -0.30 & -0.38 \\
\hline$\%$ Trials $1 \mathrm{MU}$ & 0.24 & 0.02 & 0.14 & 0.22 & 0.43 & 0.16 & 0.18 \\
\hline$\%$ Transport MU & 0.32 & 0.12 & 0.28 & 0.34 & 0.45 & 0.31 & 0.31 \\
\hline \multicolumn{8}{|l|}{ Children with $\mathrm{Bi}-\mathrm{CP}$} \\
\hline Reaching duration & 0.07 & 0.01 & 0.11 & 0.14 & 0.07 & -0.06 & 0.01 \\
\hline Peak velocity & 0.37 & 0.46 & 0.33 & 0.48 & 0.26 & -0.33 & 0.41 \\
\hline
\end{tabular}

Values are marked in bold when the relation between the kinematic parameter and the PEDI score was statistically significant $(p<0.05)$. Age groups are pooled. 
difference in gestational age at birth might have contributed to the differences between the groups. Others have demonstrated that postural control and reaching of young preterm children without $\mathrm{CP}$ differs from that of typically developing children (27-29). The present finding that quality of reaching was related to the severity of $\mathrm{CP}$, the severity of brain lesion, and the degree of spasticity, whereas such relationships were absent in the aforementioned studies, suggests that the major part of the reported differences can be attributed to the presence of $\mathrm{CP}$. Also, the finding that the degree of prematurity did not affect the kinematic parameters when the severity of brain lesion was taken into account suggests that the present findings are related mainly to the presence of CP.

Like others (4-10) we found that in typically developing children, reaching movements become faster, straighter, and smoother with increasing age. In the children with $\mathrm{SH}$, age had a limited effect on reaching parameters; in the children with $\mathrm{Bi}-\mathrm{CP}$, age did not affect the kinematics of reaching. The limited or absent effect of age can be explained in three ways. First, the absent effect of age in the group of children with $\mathrm{Bi}-\mathrm{CP}$ could be explained by the fact that the older children with Bi-CP more often had a more severe disorder than the younger children with Bi-CP (Table 2). Second, the finding in the Bi-CP group that function did not improve with age might reflect that the phenomenon of "growing into a deficit" (30) holds true not only for the most impaired limbs but also for the least impaired limb. Third, the limited improvement of reaching quality with increasing age in the children with $\mathrm{CP}$ might reflect the increased need for trial-and-error training allowing them to select the most efficient reaching strategy (31).

Our study indicated that the kinematic quality of reaching movements of the least affected arm in children with SH was worse than that of age-matched typically developing control subjects. They less often showed reaching movements of one MU, which suggests that children with SH less often relied on feedforward programming of reaching than typically developing children. They more often used the effective but more time-consuming feedback control strategy. Others also reported that the motor difficulties in children with $\mathrm{SH}$ are not restricted to the most impaired arm and hand but also involves the least impaired limb (32-34). The present finding that the impaired motor control of the dominant arm is related to the capacity to carry out daily life activities points to the clinical relevance of this finding: therapeutic intervention should pay attention not only to the most affected arm but also to the contralateral one. It should be realized that functional performance during daily life activities not only is determined by the quality of reaching of the dominant hand but also is affected by other factors such as the impairments in other parts of the body and by cognitive function (35).

The quality of reaching of the children with Bi-CP, who in general exhibited the classical pattern of spastic diplegia (24), was more severely affected than that of the children with SH. In part, this difference can be attributed to the fact that children with Bi-CP more often had a severe motor disorder, a condition that was related to a worse quality of reaching. Some of the children with severe CP received external postural support. Remarkably, this additional support did not improve the qual- ity of reaching. On the contrary, the presence of additional postural support was associated with slower reaching movements. Two explanations can be offered for this finding. The slower reaching movements could be a reflection of the severity of the motor disorder. This suggestion is supported by the finding that the children who had severe $\mathrm{CP}$ and received external support had lower PEDI scores than the children who had severe $\mathrm{CP}$ and performed testing without this additional support (24). The slower reaching movements also could be a compensatory strategy, as the reactive forces associated with slower reaching movements evoke less truncal instability (36).

In the children with $\mathrm{Bi}-\mathrm{CP}$, a higher degree of spasticity as measured by the modified Ashworth scale was related to a worse quality of reaching. However, when the effects of both the degree of spasticity and the severity of the brain lesion on the neonatal ultrasound scan were taken into account, no significant effect of the degree of spasticity was left. This means that it is the severity of brain lesion-which might be regarded as a marker of the degree of central disorganization in the nervous system-rather than the degree of spasticity that affects the quality of reaching. For clinical guidance, this means that emphasis in treatment should be on active and functional training (37) and not on the treatment of spasticity.

\section{CONCLUSION}

In conclusion, the quality of reaching of the dominant hand in children who have $\mathrm{SH}$ and $\mathrm{Bi}-\mathrm{CP}$ and are aged $2-11$ y is impaired. The impairment is related in particular to the severity of brain lesion and less to the degree of spasticity of the reaching arm or gestational age at birth. The finding that nonoptimal performance of the least impaired limbs (i.e. the least impaired arm in children with $\mathrm{SH}$ and one of the upper extremities in children with $\mathrm{Bi}-\mathrm{CP}$ ) is clearly related to functional performance in daily life indicates that therapeutic guidance should not focus solely on the most impaired limbs. Our data suggest that also functional improvement can be gained by paying attention to the function of the least impaired limb.

Acknowledgments. We thank Prof. Dr. O.F. Brouwer for critical and valuable remarks on a previous draft of this manuscript. L.A. van Eykern, A.A. Kingma-Balkema, and Dr. J.J.A.M. Schaap are kindly acknowledged for skillful technical assistance.

\section{REFERENCES}

1. Jeannerod M 1984 The timing of natural prehension movements. J Mot Behav $16: 235-254$

2. Von Hofsten C 1979 Development of visually directed reaching: the approach phase. J Hum Mov St 5:160-178

3. Fallang B, Saugstad OD, Hadders-Algra M 2000 Goal directed reaching and postural control in supine position in healthy infants. Behav Brain Res 115:9-18

4. Von Hofsten C 1991 Structuring of early reaching movements: a longitudinal study. J Mot Behav 23:280-292

5. Konczak J, Borutta M, Topka H, Dichgans J 1995 The development of goal-directed reaching in infants: hand trajectory formation and joint torque control. Exp Brain Res 106:156-168

6. Konczak J, Dichgans J 1997 The development toward stereotypic arm kinematics during reaching in the first 3 years of life. Exp Brain Res 117:346-354

7. Kuhtz-Buschbeck JP, Stolze H, Jöhnk K, Boczek-Funcke A, Illert M 1998 Development of prehension movements in children: a kinematic study. Exp Brain Res $122: 424-432$ 
8. Kuhtz-Buschbeck JP, Boczek-Funcke A, Illert M, Joehnk K, Stolze H 1999 Prehension movements and motor development in children. Exp Brain Res 128:65-68

9. Schneiberg S, Sveistrup H, McFadyen B, McKinley P, Levin MF 2002 The development of coordination for reach-to-grasp movements in children. Exp Brain Res 146:142-154

10. Van der Heide JC, Otten B, Van Eykern LA, Hadders-Algra M 2003 Developmen of postural adjustments during reaching in sitting children. Exp Brain Res $151: 32-45$

11. Utley A, Sugden D 1998 Interlimb coupling in children with hemiplegic cerebra palsy during reaching and grasping at speed. Dev Med Child Neurol 40:396-404

12. Volman MJ, Wijnroks A, Vermeer A 2002 Effect of task context on reaching performance in children with spastic hemiparesis. Clin Rehabil 16:684-692

13. Steenbergen B, Hulstijn W, de Vries A, Berger M 1996 Bimanual movemen coordination in spastic hemiparesis. Exp Brain Res 110:91-98

14. Steenbergen B, Hulstijn W, Lemmens IH, Meulenbroek RG 1998 The timing of prehensile movements in subjects with cerebral palsy. Dev Med Child Neurol 40:108-114

15. Steenbergen B, Van Thiel E, Hulstijn W, Meulenbroek RG 2000 The coordination of reaching and grasping in spastic hemiparesis. Hum Mov Sci 19:75-105

16. Van Thiel E, Steenbergen B 2001 Shoulder and hand displacements during hitting, reaching, and grasping movements in hemiparetic cerebral palsy. Motor Contro 5:166-182

17. Kluzik J, Fetters L, Coryell J 1990 Quantification of control: a preliminary study of effects of neurodevelopmental treatment on reaching in children with spastic cerebral palsy. Phys Ther 70:65-78

18. Fetters L, Kluzik J 1996 The effects of neurodevelopmental treatment versus practice on the reaching of children with spastic cerebral palsy. Phys Ther 76:346-358

19. Bohannon RW, Smith MB 1987 Interrater reliability of a modified Ashworth scale of muscle spasticity. Phys Ther 67:206-207

20. Custers JW, Wassenberg-Severijnen JE, Van der Net J, Vermeer A, Hart HT, Helder PJ 2002 Dutch adaptation and content validity of the "Pediatric Evaluation of Disability Inventory (PEDI)." Disabil Rehabil 24:250-258

21. Palisano R, Rosenbaum P, Walter S, Russell D, Wood E, Galuppi B 1997 Development and reliability of a system to classify gross motor function in children with cerebral palsy. Dev Med Child Neurol 39:214-223

22. Levene MI, Fawer CL, Lamont RF 1982 Risk factors in the development of intraventricular haemorrhage in the preterm neonate. Arch Dis Child 57:410-417

23. De Vries L, Eken P, Dubowitz LM 1992 The spectrum of leukomalacia using cranial ultrasound. Behav Brain Res 49:1-6
24. van der Heide JC, Begeer C, Fock JM, Otten E, Stremmelaar E, Van Eykern LA, Hadders-Algra M 2004 Postural control during reaching in preterm children with cerebral palsy. Dev Med Child Neurol 46:253-266

25. Otten E 2000 DataMonster, a software package running on an Apple Macintosh computer for signal analysis and modelling for research in motor control. In: Winters JM, Crago PE (eds) Biomechanics and Neural Control of Movement and Posture. Springer-Verlag, New York, pp 665-667

26. Messier J, Adamovich S, Berkinblit M, Tunik E, Poizner H 2003 Influence of movement speed on accuracy and coordination of reaching movement to memorized targets in three-dimensional space in a deafferented subject. Exp Brain Res 150:399-416

27. Hadders-Algra M, Brogren E, Katz-Salamon M, Forssberg H 1999 Periventricular leucomalacia and preterm birth have different detrimental effects on postural adjustments. Brain 122:727-740

28. van der Fits IB, Flikweert ER, Stremmelaar EF, Martijn A, Hadders-Algra M 1999 Development of postural adjustments during reaching in preterm infants. Pediatr Res 46:1-7

29. Fallang B, Saugstad OD, Grøgaard J, Hadders-Algra M 2003 Kinematic quality of reaching movements in preterm infants. Pediatr Res 53:836-842

30. Uvebrant P 1988 Hemiplegic cerebral palsy. Aetiology and outcome. Acta Paediatr Scand Suppl 345:1-100

31. Hadders-Algra M 2000 The neuronal group selection theory: promising principles for the understanding and treating of developmental motor disorders. Dev Med Child Neurol 42:707-715

32. Mercuri E, Jongmans M, Bouza H, Haataja L, Rutherford M, Henderson S, Dubowitz L 1999 Congenital hemiplegia in children at school age: assessment of hand function in the non-hemiplegic hand and correlation with MRI. Neuropediatrics 30:8-13

33. Gordon AM, Charles J, Duff SV 1999 Fingertip forces during object manipulation in children with hemiplegic cerebral palsy. II: bilateral coordination. Dev Med Child Neurol 41:176-185

34. Gordon AM, Lewis SR, Eliasson A-C, Duff SV 2003 Object release under varying task constraints in children with hemiplegic cerebral palsy. Dev Med Child Neuro 45:240-248

35. Custers JW, van der Net J, Hoijtink H, Wassenberg-Severijnen JE, Vermeer A Helders PJ 2002 Discriminative validity of the Dutch Pediatric Evaluation of Disability Inventory. Arch Phys Med Rehabil 83:1437-1441

36. Latash ML, Anson JG 1996 What are "normal movements" in atypical populations? Behav Brain Sci 19:55-68

37. Ketelaar M, Vermeer A, Hart H, van Petegem-van Beek E, Helders PJ 2001 Effects of functional therapy program on motor abilities of children with cerebral palsy. Phys Ther 81:1534-1545 